\title{
Disrupted Glutamatergic Transmission in Prefrontal Cortex Contributes to Behavioral Abnormality in an Animal Model of $\mathrm{ADHD}$
}

\author{
Jia Cheng', Aiyi Liu', Michael Y Shi' and Zhen Yan", \\ 'Department of Physiology and Biophysics, School of Medicine and Biomedical Sciences, State University of New York at Buffalo, Buffalo, \\ NY, USA
}

\begin{abstract}
Spontaneously hypertensive rats (SHR) are the most widely used animal model for the study of attention deficit hyperactivity disorder (ADHD). Here we sought to reveal the neuronal circuits and molecular basis of ADHD and its potential treatment using SHR. Combined electrophysiological, biochemical, pharmacological, chemicogenetic, and behavioral approaches were utilized. We found that AMPARmediated synaptic transmission in pyramidal neurons of prefrontal cortex (PFC) was diminished in SHR, which was correlated with the decreased surface expression of AMPAR subunits. Administration of methylphenidate (a psychostimulant drug used to treat ADHD), which blocks dopamine transporters and norepinephrine transporters, ameliorated the behavioral deficits of adolescent SHR and restored AMPAR-mediated synaptic function. Activation of PFC pyramidal neurons with a CaMKII-driven Gq-coupled designer receptor exclusively activated by designer drug also led to the elevation of AMPAR function and the normalization of ADHD-like behaviors in SHR. These results suggest that the disrupted function of AMPARs in PFC may underlie the behavioral deficits in adolescent SHR and enhancing PFC activity could be a treatment strategy for ADHD.

Neuropsychopharmacology (2017) 42, 2096-2104; doi:I0.1038/npp.2017.30; published online 22 March 2017
\end{abstract}

\section{INTRODUCTION}

Attention deficit hyperactivity disorder (ADHD) is a common neurodevelopmental disorder characterized by inattention, hyperactivity, and impulsivity. In the United States, ADHD affects about $9.0 \%$ of school-aged children (Biederman, 2005). ADHD continues into adulthood in up to half of diagnosed cases, which is often associated with depression, anxiety, and substance abuse (Biederman, 2004).

ADHD is believed to result from abnormalities in prefrontal cortex (PFC) and associated subcortical structures (Prince, 2008; Arnsten, 2009; Arnsten and Rubia, 2012). PFC is a brain region critical for 'high-level' executive functions, including working memory, sustained attention, decisionmaking, and emotional control (Goldman-Rakic, 1995, Davidson, 2002; Dalley et al, 2004; Adhikari et al, 2009). Lesion of PFC increases locomotor activity and impairs cognitive processes (Bubser and Schmidt, 1990). Delayed maturation of PFC, hypoactivity of PFC, and altered frontostriatal connection have been found in individuals with ADHD (Cortese et al, 2012; Fernández et al, 2009). The major cellular constituents in the PFC are the glutamatergic

* Correspondence: Dr Z Yan, Department of Physiology and Biophysics, State University of New York at Buffalo, 124 Sherman Hall, 3435 Main Street, Buffalo, NY I42I4, USA, Tel: 716829 3058, Fax: 7I 68292344 , E-mail: zhenyan@buffalo.edu

Received 25 October 2016; revised 23 January 2017; accepted 31 January 2017; accepted article preview online 8 February 2017 pyramidal neurons. Glutamatergic neurotransmission is pivotal for PFC-dependent functions (Goldman-Rakic, 1995; Lisman et al, 1998). Disturbed glutamate signaling in PFC has been implicated in several mental disorders, including schizophrenia and autism (Kantrowitz and Javitt, 2012; Duffney et al, 2015).

Previous studies have attributed the pathophysiological mechanism of ADHD to disturbances of the catecholamine system (Prince, 2008). The psychostimulant methylphenidate $(\mathrm{MPH})$, which increases dopamine and noradrenaline concentration in the brain (Biederman, 2005), effectively improves cognitive function and reduces hyperactivity in people with ADHD (Berridge et al, 2006). However, imaging studies of children and adults with ADHD have revealed increased levels of glutamate/glutamine in PFC and striatum (Moore et al, 2006). Emerging evidence suggests that psychostimulants also target glutamate receptors in PFC neurons (Cheng et al, 2014; Urban et al, 2013). Thus, dysfunctional glutamate system in PFC may be a key contributor to ADHD phenotypes.

The spontaneously hypertensive rats (SHRs) are the bestvalidated and most widely used animal model of ADHD (Sagvolden, 2000; Sagvolden et al, 2009). Previous studies have shown that SHR display key behavioral characteristics of ADHD, including hyperactivity, disrupted sustained attention, and impulsivity (Sagvolden et al, 2009; Russell, 2011). Aberrant dopamine signaling has been reported in SHR (Leo et al, 2003); however, the alteration of glutamate 
receptors in SHR is unclear. In this study, we have discovered the diminished AMPAR function in PFC of SHR, which can be restored by the administration of a clinically relevant dose of MPH. Moreover, activating Gq signaling to stimulate PFC pyramidal neurons using the designer receptor exclusively activated by designer drug (DREADD) technology also enhanced AMPAR function and alleviated ADHD-like behaviors in SHR. Taken together, our results provide a potential molecular basis and treatment strategy for ADHD.

\section{MATERIALS AND METHODS}

\section{Animals and Reagents}

SHRs and control Wistar-Kyoto (WKY) rats or SpragueDawley (SD) rats were obtained from Charles River Laboratories and bred at the animal facility of the State University of New York at Buffalo. Male rats (4-5 weeks old) were group housed with ad libitum access to food and water. All animal experiments were conducted with the approval of the Institutional Animal Care and Use Committee of the State University of New York at Buffalo. WKY rats were initially used as controls to compare with SHR. However, WKY rats from later breeding showed obvious problems with the growth and coordinated movement; thus, SD rats were used as controls to compare with SHR treated with $\mathrm{MPH}$ or DREADD. MPH was purchased from SigmaAldrich (St Louis, MO). Stocks were made up by dissolving in saline and stored at $-20^{\circ} \mathrm{C}$.

\section{Viral Vectors and Animal Surgery}

rAAV8-CaMKII $\alpha$-HA-hM3D(Gq)-mCherry $\left(3.8 \times 10^{12} \mathrm{vp} / \mathrm{ml}\right)$ was obtained from the UNC Vector Core (University of North Carolina, Chapel Hill, NC). The $\mathrm{hM} 3 \mathrm{D}(\mathrm{Gq})$ virus $(1 \mu \mathrm{l})$ was injected in the prelimbic region of PFC to infect glutamatergic neurons, as we described before (Yuen et al, 2012; Wei et al, 2016). In brief, rats were anesthetized and placed on a stereotaxic apparatus (David Kopf Instruments, Tujunga, CA). The injection was carried out with a Hamilton syringe (needle gauge 31 ) at a speed of $\sim 0.2 \mu \mathrm{l} / \mathrm{min}$ and the needle was kept in place for an additional $5 \mathrm{~min}$. The virus was delivered bilaterally to the PFC using the following coordinates: $2.5 \mathrm{~mm}$ anterior to the bregma, $0.75 \mathrm{~mm}$ lateral and $3.5 \mathrm{~mm}$ dorsal to ventral. Animals were allowed to recover for 2 days and used for experiments 2 weeks later. Clozapine $\mathrm{N}$-oxide $(\mathrm{CNO})$ or saline injection (i.p.) was given $1 \mathrm{~h}$ before the start of behavioral testing or animal killing, for electrophysiological recordings.

\section{Electrophysiological Recordings}

Recordings of evoked synaptic currents in prefrontal cortical slices used standard whole-cell voltage-clamp technique as we described previously (Yuen et al, 2012; Cheng et al, 2014).

Rats (4-5 weeks old) were killed after inhaling isoflurane (1-3\%). Brains were immediately removed, iced in the sucrose artificial cerebrospinal fluid (ACSF, in mM: 234 sucrose, $26 \mathrm{NaHCO}_{3}, 3 \mathrm{KCl}, 5 \mathrm{MgCl}_{2}, 1.25 \mathrm{NaH}_{2} \mathrm{PO}_{4}$, $1 \mathrm{CaCl}_{2}, 10$ glucose, $\left.\mathrm{pH} 7.4,300 \mathrm{mOsm}\right)$. Coronal slices $(300 \mu \mathrm{m})$ were obtained with a Vibratome (Leica VP1000S, Leica Microsystems, Buffalo Grove, IL). Slices were then incubated at room temperature in ACSF (in mM: $130 \mathrm{NaCl}$, $26 \mathrm{NaHCO}_{3}, 3 \mathrm{KCl}, 5 \mathrm{MgCl}_{2}, 1.25 \mathrm{NaH}_{2} \mathrm{PO}_{4}, 1 \mathrm{CaCl}_{2}, 10$ glucose, $\mathrm{pH} 7.4,300 \mathrm{mOsm}$ ) bubbled with $95 \% \mathrm{O}_{2}, 5 \% \mathrm{CO}_{2}$. Cortical slices were positioned in a perfusion chamber attached to the fixed stage of an upright Olympus microscope and submerged in continuously flowing oxygenated ACSF. Bicuculline $(10 \mu \mathrm{M})$ and CNQX $(25 \mu \mathrm{M})$ were added in NMDAR-mediated excitatory postsynaptic current (EPSC) recordings. Bicuculline and D-APV $(25 \mu \mathrm{M})$ were added in AMPAR-mediated EPSC recordings. Patch electrodes contained internal solution (in $\mathrm{mM}$ : 130 Cs-methanesulfonate, $10 \mathrm{CsCl}, 4 \mathrm{NaCl}, 10 \mathrm{HEPES}, 1 \mathrm{MgCl}_{2}$, 5 EGTA, 2.2 QX-314, 12 phosphocreatine, $5 \mathrm{MgATP}, 0.2 \mathrm{Na}_{2} \mathrm{GTP}, 0.1$ leupeptin, $\mathrm{pH}$ 7.2-7.3 and 265-270 mOsm). Cells were visualized with a $40 \times$ water-immersion lens and illuminated with near-infrared light and the image was detected with an infrared-sensitive CCD camera. A Multiclamp 700A amplifier (Molecular Device, Sunnyvale, CA) was used. Tight seals from visualized neurons were obtained by applying negative pressure. With additional suction, the membrane was disrupted into the whole-cell configuration. Evoked EPSCs were generated with a pulse from a stimulation isolation unit controlled by a S48 pulse generator (Astro-Med, West Warwick, RI). A bipolar stimulating electrode (FHC, Bowdoinham, ME) was placed $\sim 100 \mu \mathrm{m}$ from the neuron under recording. Membrane potential was maintained at $-70 \mathrm{mV}$ for AMPAR-EPSC recordings. For NMDAREPSC, cells (clamped at $-70 \mathrm{mV}$ ) were depolarized to $+60 \mathrm{mV}$ for $3 \mathrm{~s}$ before stimulation, to fully relieve the voltage-dependent $\mathrm{Mg}^{2+}$ block. To obtain the input/output responses, EPSC was elicited by a series of stimulation intensities $(50-90 \mu \mathrm{A})$ with the same duration of pulses. Miniature EPSC (mEPSC) was recorded (held at $-70 \mathrm{mV}$ ) in the low- $\mathrm{Mg}^{2+}(1 \mathrm{mM} \mathrm{MgCl})$ ACSF containing TTX $(1 \mu \mathrm{M})$.

\section{Biochemical Measurement of Surface and Total Proteins}

Surface and total proteins of AMPARs and NMDARs were detected as described previously (Yuen et al, 2012; Cheng et al, 2014). In brief, after treatment, rat cortical slices were incubated with PBS containing $1 \mathrm{mg} / \mathrm{ml}$ Sulfo-NHS-LCbiotin (Pierce Biotechnology, Rockford, IL) for $20 \mathrm{~min}$ on ice. Slices were then rinsed three times in Tris-buffered saline to quench the biotin reaction, followed by homogenization in modified radioimmunoprecipitation assay buffer (1\% Triton $\mathrm{X}-100,0.1 \%$ SDS, $0.5 \%$ deoxycholic acid, $50 \mathrm{mM} \mathrm{Na} \mathrm{PO}_{4}$, $150 \mathrm{mM} \mathrm{NaCl}, 2 \mathrm{mM}$ EDTA, $50 \mathrm{mM} \mathrm{NaF}, 10 \mathrm{mM}$ sodium pyrophosphate, $1 \mathrm{mM}$ sodium orthovanadate, $1 \mathrm{mM}$ phenylmethylsulfonyl fluoride, and $1 \mathrm{mg} / \mathrm{ml}$ leupeptin). The homogenates were centrifuged at $14000 \mathrm{~g}$ for $15 \mathrm{~min}$ at $4{ }^{\circ} \mathrm{C}$. Protein $(15 \mu \mathrm{g})$ was removed to measure the total level. For surface protein, $150 \mu \mathrm{g}$ of protein was incubated with $100 \mu \mathrm{l}$ of $50 \%$ NeutrAvidin Agarose overnight at $4{ }^{\circ} \mathrm{C}$. Proteins bound to beads were re-suspended in $100 \mu \mathrm{l}$ of $2 \times$ loading buffer, boiled and be ready detection. Protein samples were separated on $7.5 \%$ acrylamide gels and transferred to nitrocellulose membranes. The blots were blocked with $5 \%$ non-fat dry milk for $1 \mathrm{~h}$ at room temperature. Antibodies used for blotting include the following: antibodies against NR2A (EMD Millipore, Billerica, MA; 07-632), NR2B (EMD Millipore; 06-600), NR1 (Cell Signaling Technology, Danvers, MA; 5704), GluR1 
(EMD Millipore; 05-855), GluR2 (EMD Millipore; MAB397), and actin (Santa Cruz Biotechnology, Dallas, TX, sc-1616). After incubation with the appropriate secondary antibodies conjugated with horseradish peroxidase (GE Healthcare, Pittsburgh, PA), signals were visualized using an enhanced chemiluminescent detection system (Thermo Fisher Scientific, Rockford, IL). Quantitative data were obtained from measurements of immoreactive bands by Image Lab Software with the ChemiDoc MP System (BioRad, Hercules, CA).

\section{Behavioral Testing}

Temporal order recognition memory (TORM) was examined as previously described (Yuen et al, 2012; Cheng et al, 2014). The task comprised two sample phases and one test trial. In each sample phase, animals were allowed to explore two identical objects for a total of $3 \mathrm{~min}$. Different objects were used for sample phases I and II, with a $1 \mathrm{~h}$ delay between the sample phases. The test trial ( 3 min duration) was given $3 \mathrm{~h}$ after sample phase II. During the test trial, an object from sample phase I and an object from sample phase II were used. The positions of the objects in the test and sample phases were counterbalanced between animals. If temporal order memory is intact, the animals will spend more time exploring the object from sample I (ie, the novel object presented less recently), compared with the object from sample II (ie, the familiar object presented more recently). Discrimination ratio, the proportion of time spent exploring novel (less recent) $v s$ familiar (recent) objects during the test trial, was calculated.

To assess locomotor activity, midline-crossing task was performed (Cheng et al, 2014). Rats were taken from their home cages and placed into the locomotion apparatus $(45 \mathrm{~cm}$ $\mathrm{L} \times 24 \mathrm{~cm} \mathrm{~W} \times 20 \mathrm{~cm} \mathrm{H}$, with a blue line drawn along the midline of the floor). Animals were allowed to explore the apparatus for $5 \mathrm{~min}$. The number of times rats crossing the midline with all four limbs was counted.

To assess anxiety, open-field test was performed as previously described (Yuen et al, 2012; Duffney et al, 2015). Animals were placed in a corner of a rectangular apparatus $(60 \mathrm{~cm} \times 80 \mathrm{~cm})$ and the amount of time spent in the center region $(25 \mathrm{~cm} \times 25 \mathrm{~cm})$ and the number of entering the center during $5 \mathrm{~min}$ was counted. Anxious animals spend less time in the center and more time in the corner of the field.

The behavioral assays were conducted in the order of midline-crossing locomotion test, open-field test, and TORM test within 1-6 h after CNO or saline injection. The interval of different assays for each animal was at least $1 \mathrm{~h}$. As these behavioral tests were short and not stressful, the outcome of subsequent tests was not influenced by previous ones.

\section{Data Analyses and Statistics}

Data analyses were performed with Clampfit (Axon Instruments, Molecular Devices), Mini Analysis Program (Synaptosoft, Leonia, NJ), Kaleidagraph (Albeck Software, Synergy Software, Reading, PA) and GraphPad Prism 6 (GraphPad Software, La Jolla, CA). All data are expressed as the mean \pm SEM. Experiments with two groups were analyzed statistically using unpaired or paired Student's $t$-tests. Experiments with more than two groups were subjected to one-way or two-way ANOVA, followed by post-hoc Tukey's tests.

\section{RESULTS}

\section{AMPAR Function in PFC is Diminished in SHR}

Adolescent SHR (4-5 weeks old) exhibit ADHD-like phenotypes (Sagvolden, 2000; Russell, 2011); thus, they were used in this study. Given the key role of PFC glutamatergic signaling in executive functions that are impaired in ADHD, we examined the potential synaptic alterations in this brain region of SHR. AMPAR- and NMDAR-mediated EPSCs were measured in PFC pyramidal neurons. A significant reduction of the input/output curves of AMPAR-EPSC was observed in SHR (Figure 1a, 35-40\% decrease, con: $n=13$ cells $/ 4$ rats, SHR: $n=14$ cells $/ 4$ rats, $\mathrm{F}_{1,25}$ (genotype) $=$ $8.8, p<0.01$, two-way rmANOVA), whereas no significant change was observed for NMDAR-EPSC in SHR (Figure 1b,

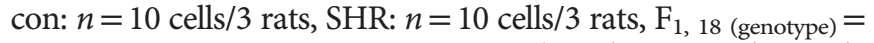
$0.06, p>0.05$, two-way rmANOVA). These results indicate that the synaptic function of AMPARs in PFC is selectively diminished in the ADHD model.

As the surface level of glutamate receptors could determine the strength of glutamatergic neurotransmission, we performed biotinylation and western blotting to examine surface expression of AMPAR and NMDAR subunits in cortical slices from SHR. As shown in Figure 1c, a significant reduction of the surface GluR1 and GluR2 subunits was observed in SHR (GluR1, 34.8\% $\pm 5.3 \%$ decrease; GluR2, $49.4 \% \pm 7.3 \%$ decrease; $n=8$ pairs, $p<0.01, t$-test). No significant change was observed for surface NR1, NR2A, and NR2B subunits in SHR ( $n=8$ pairs, $p>0.05, t$-test). Total protein levels of AMPAR and NMDAR subunits remained unchanged in SHR (Figure $1 \mathrm{~d}, n=8$ pairs, $p>0.05$, $t$-test). It suggests that the reduced surface expression of AMPAR subunits may contribute to the diminished AMPAR synaptic function in the ADHD model.

\section{MPH Rescues Behavioral Deficits and Restores AMPAR Function in SHR}

Next, we would like to understand whether the reduced AMPAR function in PFC may account for the ADHD-like behavioral deficits in SHR. MPH, a psychostimulant drug for ADHD treatment (Biederman, 2005), was used to examine the behavioral and physiological rescue in SHR.

First, we examined the impact of MPH on TORM, a cognitive process controlled by the PFC (Barker et al, 2007; Yuen et al, 2012). As shown in Figure 2a, TORM was significantly impaired in SHR, which was rescued by a single administration of the clinically relevant low-dose $\mathrm{MPH}$ $(0.5 \mathrm{mg} / \mathrm{kg}$, i.p., Berridge et al, 2006), whereas MPH did not affect TORM in control rats (discrimination ratio, control +saline: $37.8 \% \pm 3.6 \%, n=10$; control $+\mathrm{MPH}: 38.7 \% \pm 8.2 \%$, $n=8$; SHR+saline: $-11.2 \% \pm 11.3 \%, n=11 ;$ SHR+MPH, $32.1 \% \pm 10.4 \%, n=9 ; \mathrm{F}_{1,34}=5.8, p<0.05$, two-way ANOVA).

Second, we examined the impact of MPH on locomotor activities. As shown in Figure 2b, locomotion was significantly increased in SHR, which was brought to the control level by $\mathrm{MPH}$ treatment (number of midline crossing, control+saline: $11.2 \pm 0.6, n=16$; control $+\mathrm{MPH}$ : $11.1 \pm 1.0$, 

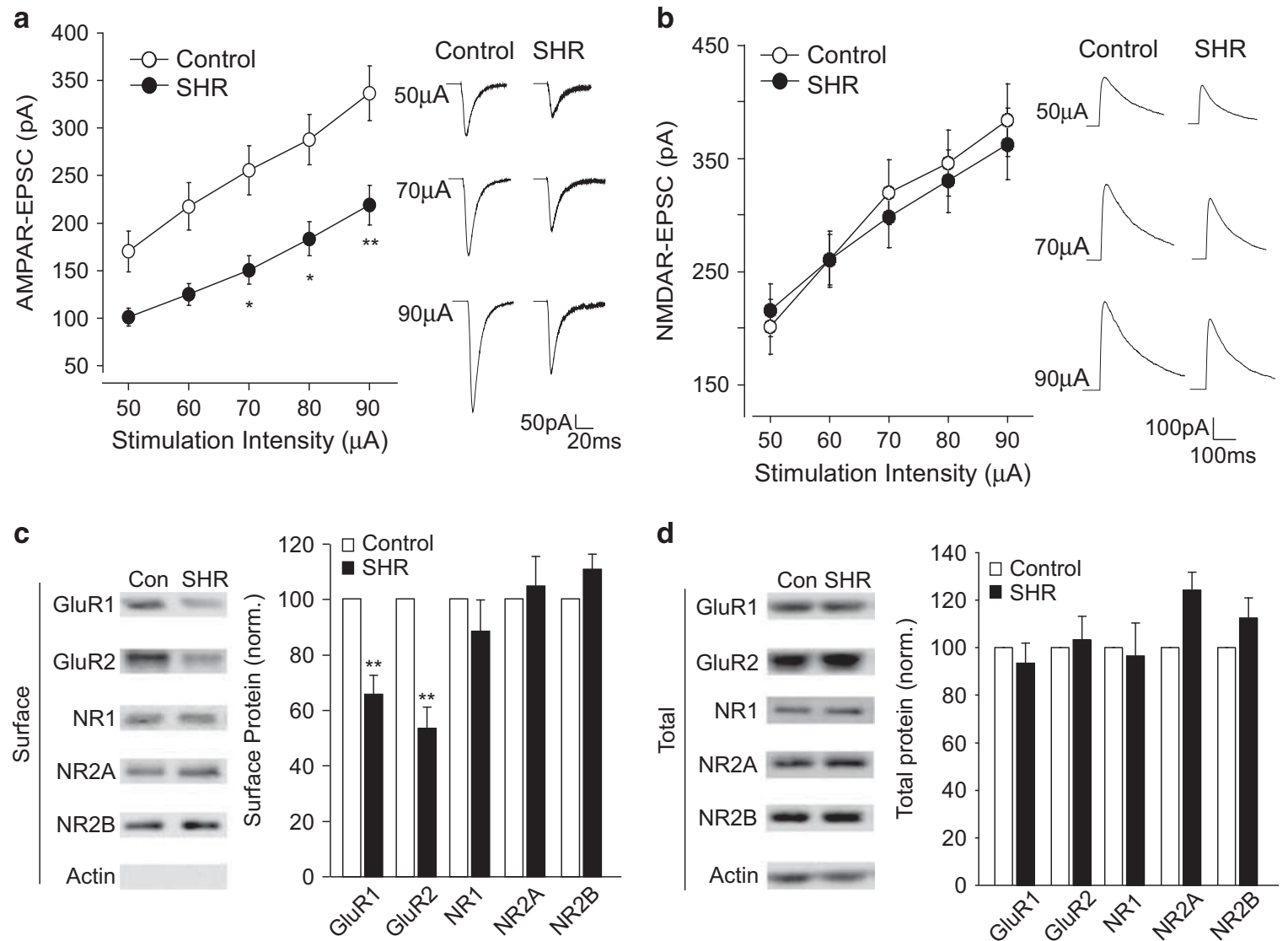

d
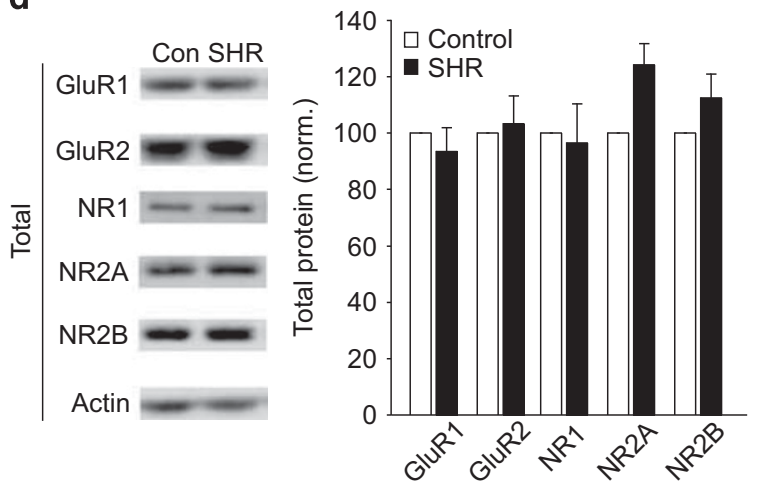

Figure I AMPAR-excitatory postsynaptic current (EPSC) and AMPAR surface expression are diminished in prefrontal cortex (PFC) pyramidal neurons of SHR. (a and b) Left: Input-output curves of AMPAR-EPSCs (a) and NMDAR-EPSCs (b) evoked by a series of stimulation intensities in PFC pyramidal neurons from control (Wistar-Kyoto, WKY) rats and spontaneously hypertensive rats (SHR). * $p<0.05$, *** $<0.0$ I, analysis of variance (ANOVA). Right: representative EPSC traces. ( $c$ and d) Immunoblots and quantification analysis of surface (c) and total (d) protein levels of AMPAR subunits and NMDAR subunits in PFC slices from control and SHR. *** $p<0.0$ I, t-test.

$n=10$; SHR+saline: $16.9 \pm 1.0, n=16$; SHR+MPH: $13.8 \pm 0.7$, $n=14 ; \mathrm{F}_{1,52}=5.2, p<0.05$, two-way ANOVA). Comparing individual SHR before and after MPH treatment, it was evident that most of these animals exhibited reduced locomotion by $\mathrm{MPH}$ (Figure 2c, $n=12, p<0.01$, paired $t$-test).

Furthermore, we examined the impact of MPH on anxiety using the open-field test. As shown in Figure 2d, MPH had little effect on control rats, whereas MPH-treated SHR became less anxious, as demonstrated by the increased time spent in the center (control+saline: $36.9 \pm 4.4 \mathrm{~s}, n=6$; control $+\mathrm{MPH}$ : $38.2 \pm 6.1 \mathrm{~s}, n=7$; SHR+saline: $16.8 \pm 6.4 \mathrm{~s}, n=10$; SHR+MPH: $42.1 \pm 5.5 \mathrm{~s}, n=8 ; \mathrm{F}_{1,27}=4.7, p<0.05$, two-way ANOVA) and increased number of center entries (control +saline: $18.7 \pm 2.5, n=6$; control+MPH: $17.1 \pm 2.5, n=7$; SHR+saline: $8.3 \pm 1.2, n=10$; SHR+MPH: $16.0 \pm 1.9, n=8$; $\mathrm{F}_{1,27}=8.5, p<0.01$, two-way ANOVA).

To complement these behavioral studies, we also examined the impact of MPH on AMPARs in SHR. As shown in Figure 3a, administration of MPH $(0.5 \mathrm{mg} / \mathrm{kg}$, i.p. $)$ significantly increased the input/output curves of AMPAR-EPSC in SHR to the level of control rats (control+saline: $n=15$ cells $/ 4$ rats; SHR+saline: $n=19$ cells/ 6 rats; SHR+MPH: $n=14$ cells/ 4 rats, $\mathrm{F}_{2,45}=9.6, p<0.001$, two-way rmANOVA). Moreover, SHR exhibited the significantly reduced amplitude and frequency of mEPSC, a synaptic response resulting from quantal release of single glutamate vesicles, which was reversed by MPH treatment (Figure 3b, control+saline: $n=21$ cells $/ 2$ rats; control+MPH: $n=17$ cells $/ 2$ rats; SHR +saline: $n=28$ cells/ 3 rats; SHR+MPH: $n=19$ cells/ 3 rats, $\mathrm{F}_{1,80}=17.1$ for amplitude, $p<0.01, \mathrm{~F}_{1,80}=3.8$ for frequency, $p<0.05$, two-way ANOVA). The restoration of AMPAR function in PFC may contribute to the behavioral normalization in SHR treated with MPH.

\section{Chemicogenetic Activation of PFC Pyramidal Neurons Elevates AMPAR Function and Normalizes Behaviors in SHR}

To further confirm that PFC hypofunction underlies the ADHD-like behavioral deficits in SHR, we used a novel strategy based on DREADDs, to remotely control PFC neuronal activity in vivo. By applying the pharmacologically inert, exogenously administered ligand CNO, DREADDs enable the activation of endogenous G-protein signaling pathways in discrete neuronal populations (Alexander et al, 2009; Wess et al, 2013; Urban and Roth, 2015). To selectively stimulate PFC pyramidal neurons, we injected a CaMKIIdriven Gq-coupled DREADD adeno-associated virus, hM3D $(\mathrm{Gq})$, into medial PFC of SHR (Figure 4a). Application of 
CNO $(5 \mu \mathrm{M})$ significantly increased the frequency of spontaneous action potentials in $\mathrm{hM} 3 \mathrm{D}(\mathrm{Gq})$-expressing PFC pyramidal neurons (Figure $4 \mathrm{~b}-\mathrm{d}$, baseline: $1.6 \pm 0.3 \mathrm{~Hz}$, +CNO: $4.3 \pm 0.2 \mathrm{~Hz}, n=7, p<0.001, t$-test), confirming the elevation of neuronal activity by this chemicogenetic approach. In hM3D(Gq)-infected SHR, the input/output curves of AMPAR-EPSC in PFC pyramidal neurons were significantly increased by the injection of CNO, but not saline (Figure $4 \mathrm{e}$ and $\mathrm{f}$, control+saline: $n=7$ cells $/ 2$ rats; control+CNO: $n=8$ cells/ 2 rats; SHR+saline: $n=18$ cells/ 3 rats; SHR+CNO: $n=20$ cells $/ 5$ rats, $\mathrm{F}_{3,49}=7.0, p<0.001$, two-way rmANOVA), suggesting that activating Gq signaling in PFC is capable of restoring AMPAR function in the ADHD model.

As DREADD-mediated activation of PFC rescued the synaptic deficits in SHR, we also examined its impact on ADHD-like behaviors. In CNO-injected SHR with PFC infection of $\mathrm{hM} 3 \mathrm{D}(\mathrm{Gq})$, the discrimination ratio in the TORM task was significantly improved (Figure 5a, control+saline: $31.6 \% \pm 5.2 \%, n=5$; control+CNO: $29.3 \% \pm 3.6 \%, n=5$; SHR +saline: $-13.4 \% \pm 10.9 \%, n=4$; $\mathrm{SHR}+\mathrm{CNO}: 22.2 \% \pm 7.5 \%$, $n=5, \mathrm{~F}_{1,15}=5.8, p<0.05$, two-way ANOVA), and the locomotor activity was significantly reduced (Figure 5b, Number of midline crossing, control+saline: $12.6 \pm 0.9, n=5$; control+CNO: $13.2 \pm 1.3, n=5$; SHR+saline: $19.2 \pm 2.2, n=4$; SHR+CNO: $12.0 \pm 0.8, \quad n=5, \mathrm{~F}_{1,15}=6.5, p<0.05$, two-way
ANOVA). These PFC-activated SHR also became less anxious, as demonstrated by the increased time spent in the center (control+saline: $31.0 \pm 3.8 \mathrm{~s}, n=5$; control+CNO: $33.8 \pm 7.1 \mathrm{~s}$, $n=5$; SHR+saline: $11.0 \pm 1.6 \mathrm{~s}, n=7$; SHR+CNO, $29.8 \pm 4.6 \mathrm{~s}$, $n=5, \mathrm{~F}_{1,18}=6.2, p<0.05$, two-way ANOVA) and increased number of center entries (control+saline: $20.2 \pm 2.4, n=5$; control+CNO: $20.4 \pm 2.8, n=5$; SHR+saline: $8.4 \pm 0.6, n=7$; SHR+CNO: $19.2 \pm 3.1, n=5, \mathrm{~F}_{1,18}=6.2, p<0.05$, two-way ANOVA) in the open-field test. No significant effects on the tested behaviors were observed in $\mathrm{hM} 3 \mathrm{D}(\mathrm{Gq})$-infected control rats with $\mathrm{CNO}$ injection. These data suggest that DREADD is effective in pathological conditions and elevating PFC activity by stimulating the $\mathrm{Gq}$ signaling in pyramidal neurons is sufficient for the behavioral normalization in the ADHD model.

\section{DISCUSSION}

Despite the prevalence of ADHD, the molecular and cellular basis of this mental disorder remains elusive. Hypoactivity of PFC has been found in individuals with ADHD (Cortese et al, 2012; Fernández et al, 2009), suggesting the importance of this brain region in controlling ADHD-associated behaviors. In this study, we have found that AMPARmediated synaptic transmission in PFC is diminished in an ADHD model, consistent with the idea that ADHD is a
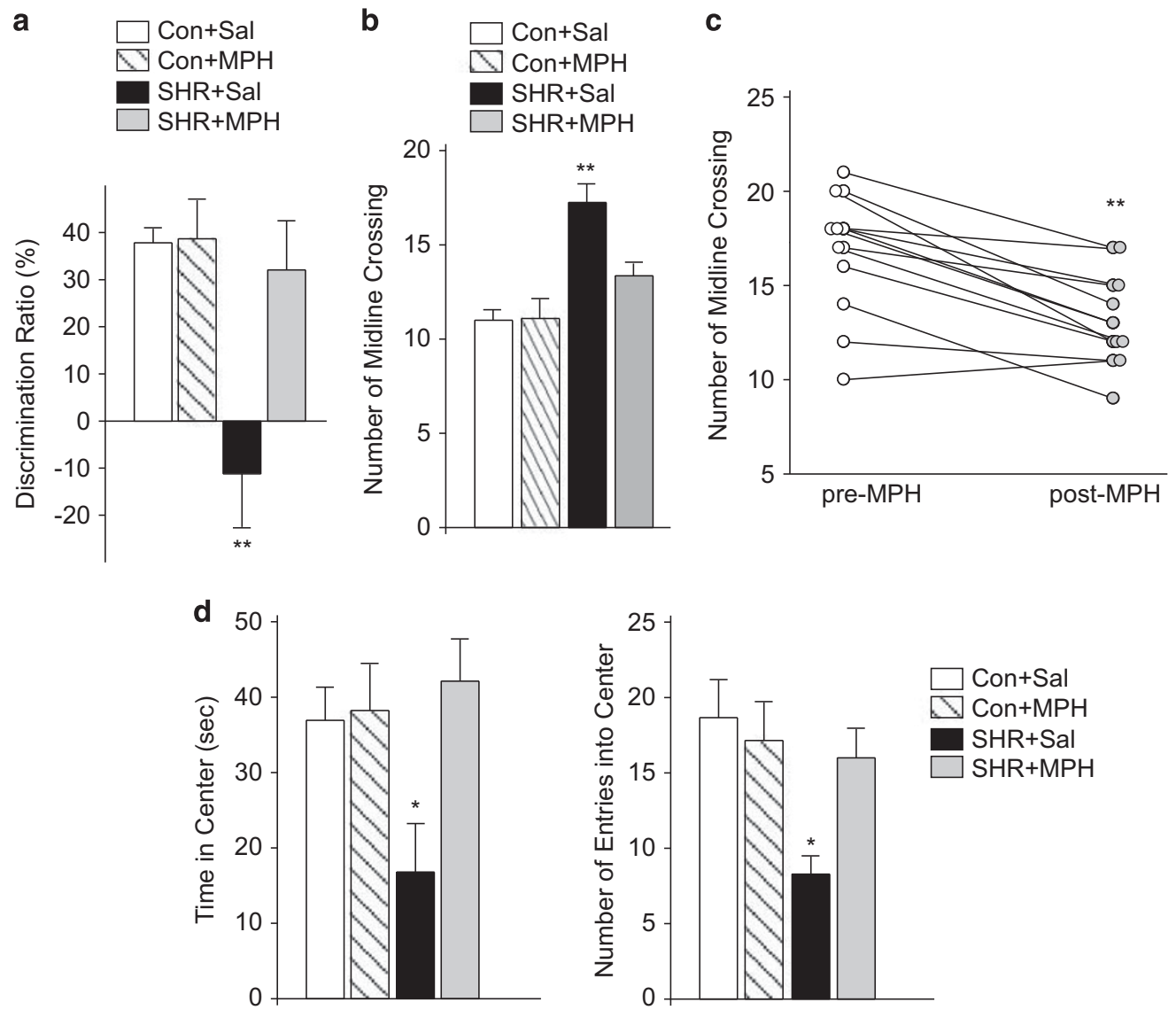

Figure 2 Methylphenidate $(\mathrm{MPH})$ treatment rescues behavioral deficits in spontaneously hypertensive rat (SHR). (a) Bar graphs (mean \pm SEM) showing the discrimination ratio of the temporal order recognition memory (TORM) task in SHR or control (Sprague-Dawley, SD) rats treated with saline or MPH ( $0.5 \mathrm{mg} / \mathrm{kg}$, i.p., I h before testing). *** $<0.0 \mathrm{I}$, analysis of variance (ANOVA). (b) Bar graphs showing the number of midline crossing in locomotion test in SHR or control rats treated with saline or MPH. $* * * 2<0.01$, ANOVA. (c) Dot plots showing the number of midline crossing in SHR before and after MPH treatment. ${ }^{*} * 0.0 \mathrm{I}$, paired t-test. (d) Bar graphs showing the time in the center and the number of center entries in the open-field test of SHR or control rats treated with saline or $\mathrm{MPH} . * 0<0.05$, ANOVA. 

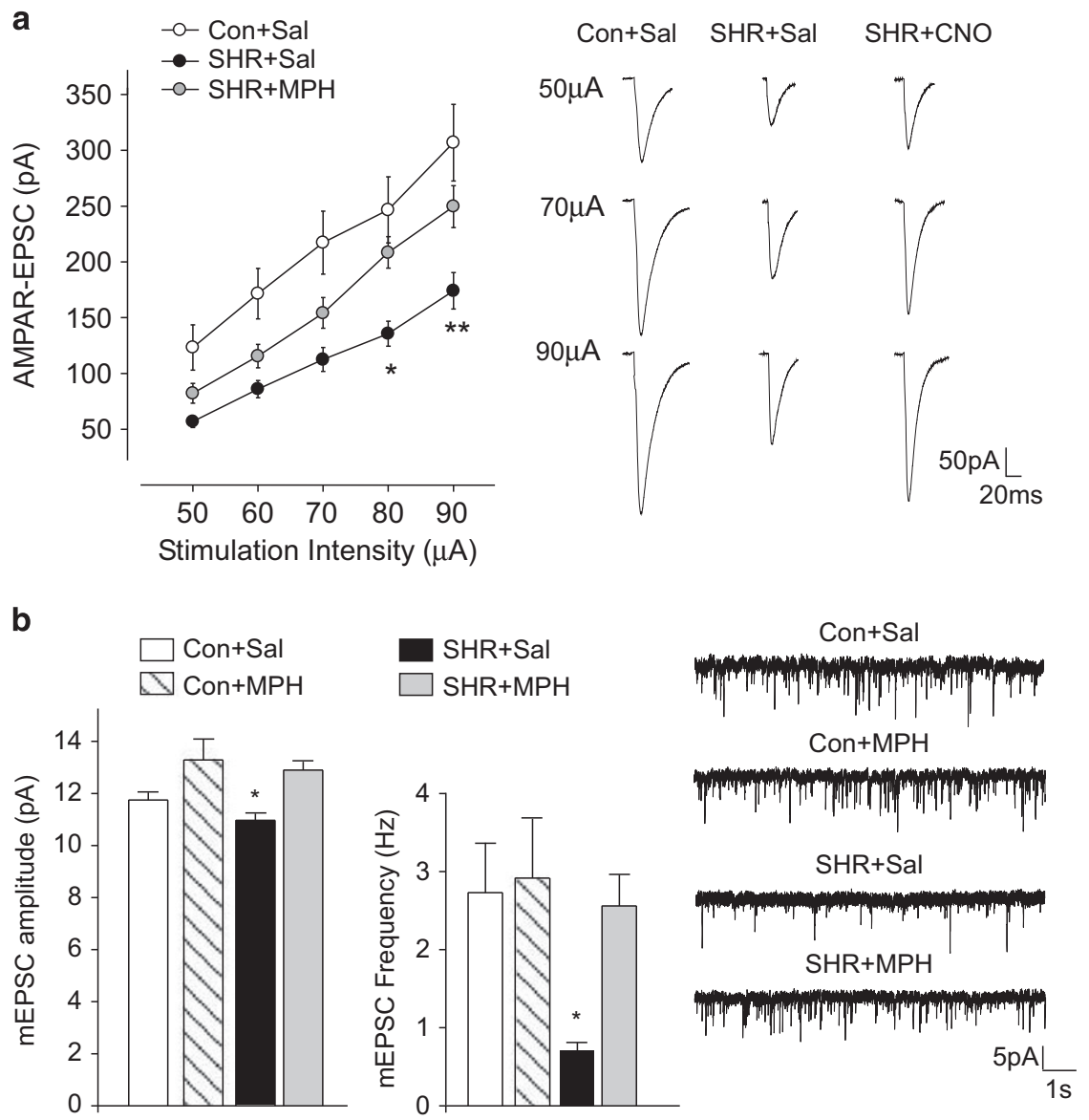

Figure 3 Methylphenidate (MPH) treatment enhances AMPAR-excitatory postsynaptic current (EPSC) in prefrontal cortex (PFC) pyramidal neurons of spontaneously hypertensive rat (SHR). (a) Left: Input-output curves of AMPAR-EPSC evoked by a series of stimulation intensities in PFC pyramidal neurons from SHR or control (Sprague-Dawley, SD) rats treated with saline or MPH $(0.5 \mathrm{mg} / \mathrm{kg})$. $* p<0.05$, *** $<0.0$ I, analysis of variance (ANOVA), compared with SHR+MPH. Right: representative EPSC traces. (b) Left: Bar graphs (mean \pm SEM) showing the miniature EPSC (mEPSC) amplitude or frequency in PFC pyramidal neurons from SHR or control rats treated with saline or $\mathrm{MPH}$. ${ }^{*} p<0.05$, ANOVA. Right: representative mEPSC traces.

hypoglutamatergic condition, with prefrontal brain regions being especially affected (Carlsson, 2001). Magnetic resonance spectroscopy studies have reported the altered glutamate to glutamine ratio in different brain regions in patients with ADHD (Carrey et al, 2007; Perlov et al, 2007). Genome-wide screening has identified the association of several genetic variation with $\mathrm{ADHD}$, including glutamate receptor polymorphism (Turic et al, 2004). AMPA receptor, the major glutamate receptor involved in synaptic transmission and cognitive functions, has been considered as a potential drug target for enhancing attention and alertness (Partin, 2015).

SHR, the most established ADHD model (Sagvolden, 2000; Sagvolden et al, 2009), has been extensively examined, especially in terms of abnormalities in dopamine and norepinephrine signaling (Russell, 2001; Leo et al, 2003; Sterley et al, 2014). The monoaminergic alterations of SHR may or may not have the involvement of glutamatergic system, particularly AMPARs. However, our direct measurement of AMPAR-mediated synaptic responses has revealed the glutamatergic deficits in PFC pyramidal neurons of SHR, probably at both pre- and post-synaptic locus. Abnormalities in AMPAR function in ADHD have been suggested to result from caspase 3 deficiency (Lo et al, 2015). The detailed underlying mechanism awaits to be further studied.

Our data on the MPH-induced rescue of ADHD-like behaviors and AMPAR function in SHR further suggest the importance of PFC glutamate signaling in the pathophysiology of ADHD. Adolescent SHR exhibits the impaired TORM, a PFC-mediated cognitive process (Barker et al, 2007; Yuen et al, 2012), suggesting PFC dysfunction. Consistently, deficits in spatial learning and working memory have been found in SHR (Nakamura-Palacios et al, 1996). Children with ADHD have deficit in working memory, which is attributed to an impairment of the frontal lobe (Smith et al, 2006). Training of working memory in children with ADHD has been suggested as a potential clinical use for ameliorating ADHD symptoms (Klingberg et al, 2002, 2005). Adolescent SHR exhibits increased locomotor activity, consistent with the previous observation of hyperactivity in adult SHR (Sagvolden et al, 2009; Russell, 2011). Interestingly, MPH treatment mitigates the working memory deficits, hyperactivity and anxiety in SHR, which correlates with the MPH-induced enhancement of AMPAR function in PFC pyramidal neurons of SHR.

$\mathrm{MPH}$ acutely and effectively alleviates cognitive dysfunction and hyperactivity in individuals with ADHD (Spencer et al, 
a
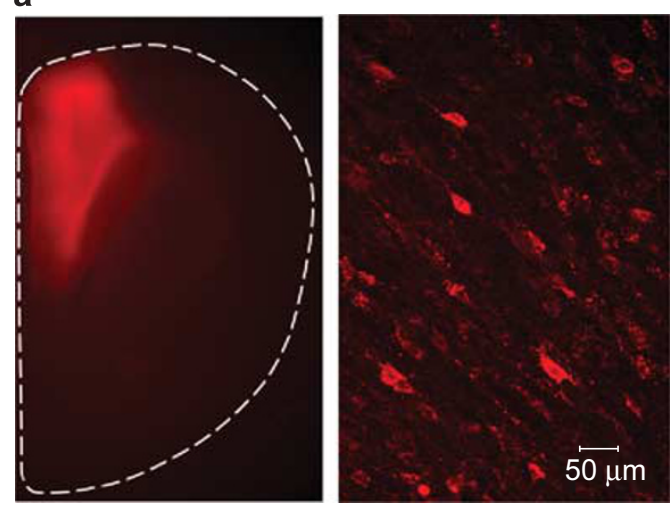

C
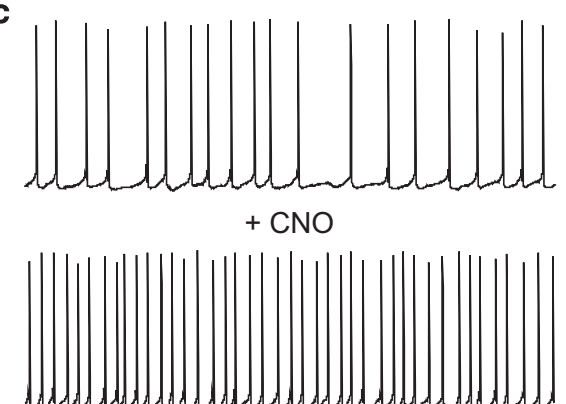

$20 \mathrm{mV}$

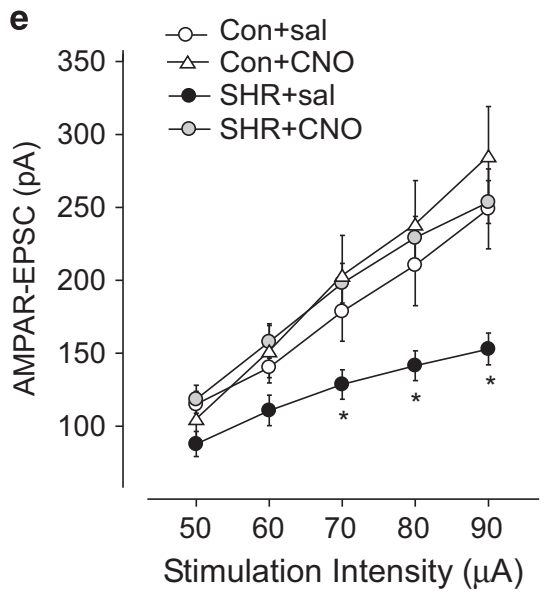

f $50 \mu \mathrm{A}$ b

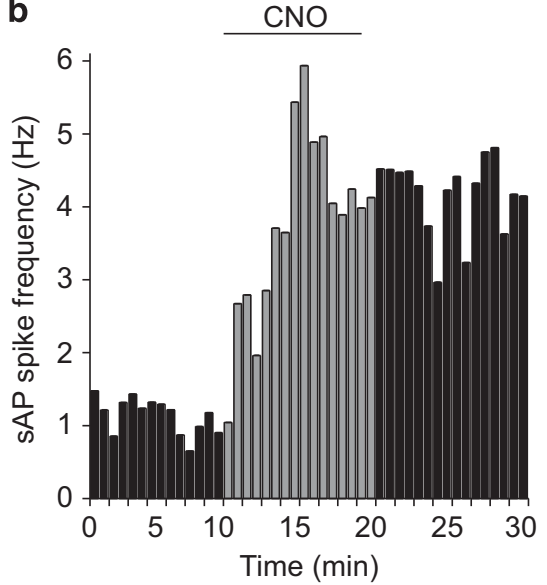

d

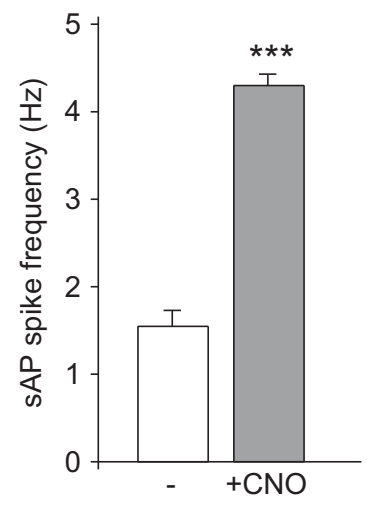

Con+Sal Con+CNO SHR+Sal SHR+CNO<smiles>CCCC</smiles><smiles>CCCC</smiles><smiles>CCCCCCCC</smiles><smiles>CCCCCCCCCCCCC</smiles><smiles>CCCC</smiles><smiles>CCCC</smiles>
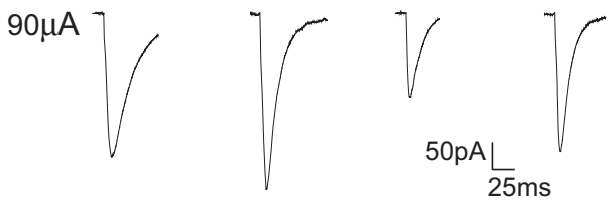

Figure 4 Chemicogenetic activation of prefrontal cortex (PFC) pyramidal neurons in spontaneously hypertensive rat (SHR) enhances AMPAR-excitatory postsynaptic current (EPSC). (a) Low- and high-magnification images showing hM3Dq adeno-associated virus (AAV) delivery to PFC pyramidal neurons. (b) Time course showing the frequencies of spontaneous AP (SAP) before and after bath application of clozapine N-oxide (CNO) $(5 \mu M)$ in a PFC pyramidal neuron from a rat with the injection of hM3Dq AAV to medial PFC. (c) Representative sAP traces before and after CNO application. (d) Bar graphs (mean \pm SEM) showing sAP frequencies in the absence $(-)$ or presence of CNO in PFC neurons from hM3Dq AAV-infected rats. **** $p<0.00 \mathrm{I}, t$-test. (e) AMPAR-EPSC I/O in PFC pyramidal neurons (hM3Dq AAV-infected) from SHR or control (Sprague-Dawley, SD) rats injected with saline or CNO. ${ }^{*} p<0.05$, analysis of variance. $f$, Representative AMPAR-EPSC traces.

1996). It is known that MPH occupies and blocks dopamine transporters and noradrenaline transporters, resulting in elevated dopamine and noradrenaline levels at synapses (Spencer et al, 2006; Kuczenski and Segal, 1997). Emerging studies indicate that glutamate system is also regulated by MPH. AMPAR surface expression in hippocampus CA1 area is increased by MPH through a PKA-dependent pathway
(Rozas et al, 2015). In naive animals, NMDARs are regulated by MPH (Cheng et al, 2014; Urban et al, 2013; Di Miceli and Gronier, 2015), which may contribute to the MPH-induced cognitive enhancement (Cheng et al, 2014). In the ADHD model, we have found that AMPARs are regulated by MPH, which may underlie the therapeutic efficacy of MPH on ADHD-associated behaviors. It is in agreement with previous 
a
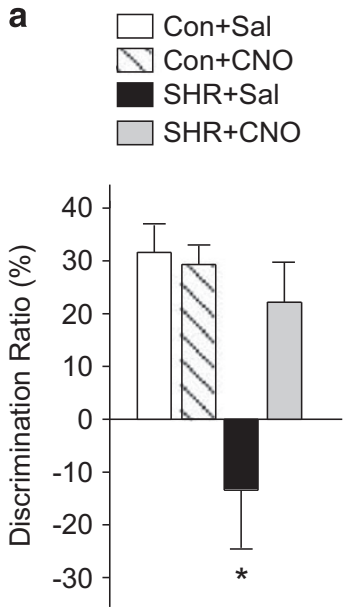

b
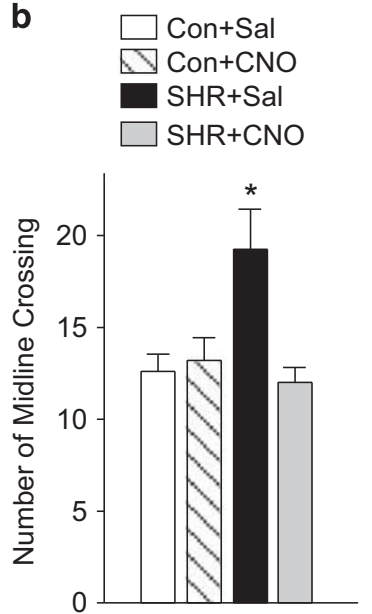

C
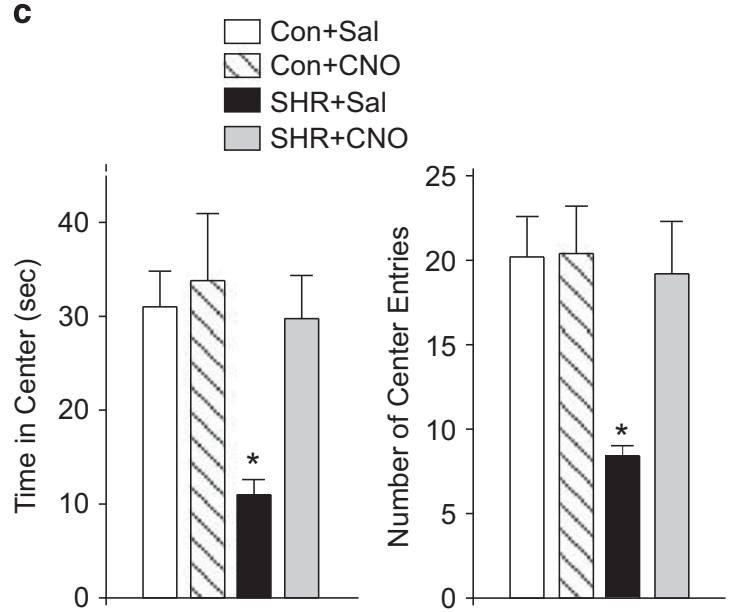

Figure 5 Chemicogenetic activation of prefrontal cortex (PFC) pyramidal neurons in spontaneously hypertensive rat (SHR) rescues behavioral deficits. (a) Bar graphs (mean \pm SEM) showing the discrimination ratio of the temporal order recognition memory (TORM) task in SHR or control (Sprague-Dawley, SD) rats (hM3Dq adeno-associated virus (AAV)-infected) injected with saline or clozapine $\mathrm{N}$-oxide (CNO). *p $<0.05$, analysis of variance (ANOVA). (b) Bar graphs showing the number of midline crossing in locomotion test in SHR or control rats (hM3Dq AAV-infected) injected with saline or CNO. *p $<0.05$, ANOVA. (c) Bar graphs showing the time in the center and the number of center entries in the open-field test of SHR or control rats (hM3Dq AAV-infected) injected with saline or CNO. $*$ p $<0.05$, ANOVA.

findings that psychostimulants work differently in normal $v s$ diseased conditions.

To further link PFC hypofunction to ADHD-like phenotypes in SHR, we have used the chemicogenetic approach to directly activate PFC pyramidal neurons by stimulating $\mathrm{Gq}$ signaling. The DREADD-based strategy allows for the precise interrogation of specific neuronal circuits involved in cognitive and emotional processes (Wess et al, 2013; Urban and Roth, 2015). By the remote, non-invasive and long-lasting manipulation of the activity of PFC pyramidal neurons in vivo, we have observed the restoration of AMPAR responses in SHR. The increase of AMPAR-EPSC inputoutput ratio by activation of $\mathrm{Gq}$ receptors with the excitatory DREADDs could be due to changes in firing rate or the induction of synaptic plasticity. Consistently, recent studies have found that activation of hippocampal pyramidal neurons with the CaMKII-driven Gq-coupled DREADD increases baseline synaptic transmission and long-term potentiation (López et al, 2016). Interestingly, elevating the activity of PFC pyramidal neurons leads to the amelioration of ADHD-like behaviors in SHR. It provides a potential approach to treat mental disorders associated with PFC network dysfunction, such as ADHD.

\section{FUNDING AND DISCLOSURE}

The authors declare no conflict of interest.

\section{ACKNOWLEDGMENTS}

We thank Xiaoqing Chen for her excellent technical support. This work was supported by NIH grants DA037618 and MH108842 to ZY.

\section{REFERENCES}

Adhikari A, Topiwala MA, Gordon JA (2009). Synchronized activity between the ventral hippocampus and the medial prefrontal cortex during anxiety. Neuron 65: 257-269.

Alexander GM, Rogan SC, Abbas AI, Armbruster BN, Pei Y, Allen JA et al (2009). Remote control of neuronal activity in transgenic mice expressing evolved $\mathrm{G}$ protein-coupled receptors. Neuron 63: 27-39.

Arnsten AF (2009). Toward a new understanding of attentiondeficit hyperactivity disorder pathophysiology: an important role for prefrontal cortex dysfunction. CNS Drugs 23: 33-41.

Arnsten AF, Rubia K (2012). Neurobiological circuits regulating attention, cognitive control, motivation, and emotion: disruptions in neurodevelopmental psychiatric disorders. J Am Acad Child Adolesc Psychiatry 51: 356-367.

Barker GR, Bird F, Alexander V, Warburton EC (2007). Recognition memory for objects, place, and temporal order: a disconnection analysis of the role of the medial prefrontal cortex and perirhinal cortex. J Neurosci 27: 2948-2957.

Berridge CW, Devilbiss DM, Andrzejewski ME, Arnsten AF, Kelley AE, Schmeichel B et al (2006). Methylphenidate preferentially increases catecholamine neurotransmission within the prefrontal cortex at low doses that enhance cognitive function. Biol Psychiatry 60: 1111-1120.

Biederman J (2004). Impact of comorbidity in adults with attention deficit/hyperactivity disorder. J Clin Psychiatry Vol 65(Suppl3): 3-7.

Biederman J (2005). Attention-deficit/hyperactivity disorder: a selective overview. Biol Psychiatry 57: 1215-1220.

Bubser M, Schmidt WJ (1990). 6-Hydroxydopamine lesion of the rat prefrontal cortex increases locomotor activity, impairs acquisition of delayed alternation tasks, but does not affect uninterrupted tasks in the radial maze. Behav Brain Res 37: 157-168.

Carlsson ML (2001). On the role of prefrontal cortex glutamate for the antithetical phenomenology of obsessive compulsive disorder and attention deficit hyperactivity disorder. Prog Neuropsychopharmacol Biol Psychiatry 25: 5-26.

Carrey NJ, MacMaster FP, Gaudet L, Schmidt MH (2007). Striatal creatine and glutamate/glutamine in attention-deficit/hyperactivity disorder. J Child Adolesc Psychopharmacol 17: 11-17. 
Cheng J, Xiong Z, Duffney LJ, Wei J, Liu A, Liu S et al (2014). Methylphenidate exerts dose-dependent effects on glutamate receptors and behaviors. Biol Psychiatry 76: 953-962.

Cortese S, Kelly C, Chabernaud C, Proal E, Di Martino A, Milham MP et al (2012). Toward systems neuroscience of ADHD: a meta-analysis of 55 fMRI studies. Am J Psychiatry 169: 1038-1055.

Dalley JW, Cardinal RN, Robbins TW (2004). Prefrontal executive and cognitive functions in rodents: neural and neurochemical substrates. Neurosci Biobehav Res 28: 771-784.

Davidson RJ (2002). Anxiety and affective style: role of prefrontal cortex and amygdala. Biol Psychiatry 51: 68-80.

Di Miceli M, Gronier B (2015). Psychostimulants and atomoxetine alter the electrophysiological activity of prefrontal cortex neurons, interaction with catecholamine and glutamate NMDA receptors. Psychopharmacology (Berl) 232: 2191-2205.

Duffney LJ, Zhong P, Wei J, Matas E, Cheng J, Qin L et al (2015). Autism-like deficits in Shank3-deficient mice are rescued by targeting actin regulators. Cell Rep 11: 1400-1413.

Fernández A, Quintero J, Hornero R, Zuluaga $\mathrm{P}$, Navas $\mathrm{M}$, Gómez C et al (2009). Complexity analysis of spontaneous brain activity in attention-deficit/hyperactivity disorder: diagnostic implications. Biol Psychiatry 65: 571-577.

Goldman-Rakic PS (1995). Cellular basis of working memory. Neuron 14: 477-485.

Kantrowitz J, Javitt DC (2012). Glutamatergic transmission in schizophrenia: from basic research to clinical practice. Curr Opin Psychiatry 25: 96-102.

Klingberg T, Fernell E, Olesen PJ, Johnson M, Gustafsson P, Dahlström $\mathrm{K}$ et al (2005). Computerized training of working memory in children with ADHD-a randomized, controlled trial. J Am Acad Child Adolesc Psychiatry 44: 177-186.

Klingberg T, Forssberg H, Westerberg H (2002). Training of working memory in children with ADHD. J Clin Exp Neuropsychol 24: 781-791.

Kuczenski R, Segal DS (1997). Effects of methylphenidate on extracellular dopamine, serotonin, and norepinephrine: comparison with amphetamine. J Neurochem 68: 2032-2037.

Leo D, Sorrentino E, Volpicelli F, Eyman M, Greco D, Viggiano D et al (2003). Altered midbrain dopaminergic neurotransmission during development in an animal model of ADHD. Neurosci Biobehav Rev. 27: 661-669.

Lisman JE, Fellous JM, Wang XJ (1998). A role for NMDA-receptor channels in working memory. Nat Neurosci 1: 273-275.

López AJ, Kramár E, Matheos DP, White AO, Kwapis J, Vogel-Ciernia A et al (2016). Promoter-specific effects of DREADD modulation on hippocampal synaptic plasticity and memory formation. J Neurosci 36: 3588-3599.

Lo SC, Wang Y, Weber M, Larson JL, Scearce-Levie K, Sheng M (2015). Caspase-3 deficiency results in disrupted synaptic homeostasis and impaired attention control. J Neurosci 35: 2118-2132.

Moore CM, Biederman J, Wozniak J, Mick E, Aleardi M, Wardrop M et al (2006). Differences in brain chemistry in children and adolescents with attention deficit hyperactivity disorder with and without comorbid bipolar disorder: a proton magnetic resonance spectroscopy study. Am J Psychiatry 163: 316-318.

Nakamura-Palacios EM, Caldas CK, Fiorini A, Chagas KD, Chagas KN, Vasquez EC (1996). Deficits of spatial learning and working memory in spontaneously hypertensive rats. Behav Brain Res 74: 217-227.

Partin KM (2015). AMPA receptor potentiators: from drug design to cognitive enhancement. Curr Opin Pharmacol 20: 46-53.

Perlov E, Philipsen A, Hesslinger B, Buechert M, Ahrendts J, Feige B et al (2007). Reduced cingulate glutamate/glutamine-to-creatine ratios in adult patients with attention deficit/hyperactivity disorder - a magnet resonance spectroscopy study. J Psychiatr Res 41: 934-941.

Prince J (2008). Catecholamine dysfunction in attention-deficit/ hyperactivity disorder: an update. J Clin Psychopharmacol 28: S39-S45.

Rozas C, Carvallo C, Contreras D, Carreño M, Ugarte G, Delgado R et al (2015). Methylphenidate amplifies long-term potentiation in rat hippocampus CA1 area involving the insertion of AMPA receptors by activation of $\beta$-adrenergic and D1/D5 receptors. Neuropharmacology 99: 15-27.

Russell VA (2001). Increased AMPA receptor function in slices containing the prefrontal cortex of spontaneously hypertensive rats. Metab Brain Dis 16: 143-149.

Russell VA (2011). Overview of animal models of attention deficit hyperactivity disorder (ADHD). Curr Protoc Neurosci Chapter 9: Unit 9.3535.

Sagvolden T (2000). Behavioral validation of the spontaneously hypertensive rat (SHR) as an animal model of attention-deficit/ hyperactivity disorder (AD/HD). Neurosci Biobehav Rev 24: 31-39.

Sagvolden T, Johansen EB, Woien G, Walaas SI, Storm-Mathisen J, Bergersen LH et al (2009). The spontaneously hypertensive rat model of $\mathrm{ADHD}$ - the importance of selecting the appropriate reference strain. Neuropharmacology 57: 619-626.

Smith AB, Taylor E, Brammer M, Toone B, Rubia K (2006). Taskspecific hypoactivation in prefrontal and temporoparietal brain regions during motor inhibition and task switching in medication-naive children and adolescents with attention deficit hyperactivity disorder. Am J Psychiatry 163: 1044-1051.

Spencer T, Biederman J, Wilens T, Harding M, O'Donnell D, Griffin S (1996). Pharmacotherapy of attention-deficit hyperactivity disorder across the life cycle. J Am Acad Child Adolesc Psychiatry 35: 409-432.

Spencer TJ, Biederman J, Ciccone PE, Madras BK, Dougherty DD, Bonab AA et al (2006). PET study examining pharmacokinetics, detection and likeability, and dopamine transporter receptor occupancy of short- and long-acting oral methylphenidate. Am J Psychiatry 163: 387-395.

Sterley TL, Howells FM, Russell VA (2014). Nicotine-stimulated release of $[3 \mathrm{H}]$ norepinephrine is reduced in the hippocampus of an animal model ofattention-deficit/hyperactivity disorder, the spontaneously hypertensive rat. Brain Res 1572: 1-10.

Turic D, Langley K, Mills S, Stephens M, Lawson D, Govan C et al (2004). Follow-up of genetic linkage findings on chromosome 16p13: evidence of association of N-methyl-D aspartate glutamate receptor 2A gene polymorphism with ADHD. Mol Psychiatry 9: 169-173.

Urban DJ, Roth BL (2015). DREADDs (designer receptors exclusively activated by designer drugs): chemogenetic tools with therapeutic utility. Annu Rev Pharmacol Toxicol. 55: 399-417.

Urban KR, Li YC, Gao WJ (2013). Treatment with a clinicallyrelevant dose of methylphenidate alters NMDA receptor composition and synaptic plasticity in the juvenile rat prefrontal cortex. Neurobiol Learn Mem 101: 65-74.

Wei J, Xiong Z, Lee JB, Cheng J, Duffney LJ, Matas E et al (2016). Histone modification of Nedd4 ubiquitin ligase controls the loss of AMPA receptors and cognitive impairment induced by repeated stress. J Neurosci 36: 2119-2130.

Wess J, Nakajima K, Jain S (2013). Novel designer receptors to probe GPCR signaling and physiology. Trends Pharmacol Sci 34: 385-392.

Yuen EY, Wei J, Liu W, Zhong P, Li X, Yan Z (2012). Repeated stress causes cognitive impairment by suppressing glutamate receptor expression and function in prefrontal cortex. Neuron 73: 962-977. 